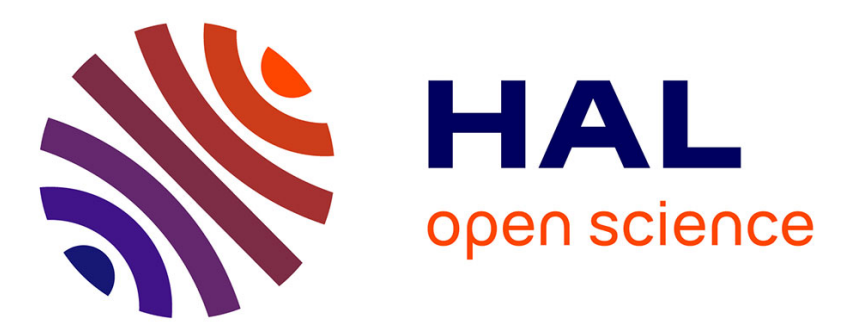

\title{
Nouvelle architecture électromagnétique à réluctance variable excitée pour accumulateur électromécanique d'énergie
}

Olivier Gergaud, Hamid Ben Ahmed, Bernard Multon, Nicolas Bernard

\section{- To cite this version:}

Olivier Gergaud, Hamid Ben Ahmed, Bernard Multon, Nicolas Bernard. Nouvelle architecture électromagnétique à réluctance variable excitée pour accumulateur électromécanique d'énergie. RIGE (Revue Internationale de Génie Electrique ), 2001, Vol.4 (n³/4), pp.367-390. hal-00674233

\section{HAL Id: hal-00674233 \\ https://hal.science/hal-00674233}

Submitted on 26 Feb 2012

HAL is a multi-disciplinary open access archive for the deposit and dissemination of scientific research documents, whether they are published or not. The documents may come from teaching and research institutions in France or abroad, or from public or private research centers.
L'archive ouverte pluridisciplinaire HAL, est destinée au dépôt et à la diffusion de documents scientifiques de niveau recherche, publiés ou non, émanant des établissements d'enseignement et de recherche français ou étrangers, des laboratoires publics ou privés. 


\title{
Nouvelle architecture électromagnétique à réluctance variable excitée pour accumulateur électromécanique d'énergie
}

\author{
Olivier Gergaud - Hamid Ben Ahmed - Bernard Multon - Nicolas \\ Bernard
}

LESiR ESA CNRS 8029

Antenne de Bretagne de L'Ecole Normale Supérieure de Cachan

Campus de Ker lann

35170 BRUZ

gergaud@bretagne.ens-cachan.fr

RÉSUMÉ. Cet article traite d'un moteur-générateur original destiné au stockage électromécanique d'énergie. Il est de type synchrone à excitation homopolaire fixe, à bobinage d'induit dans l'entrefer et d'architecture discoïde pour une meilleure intégration au volant d'inertie. Il ne doit pas perturber les suspensions magnétiques du dispositif, pour cela nous avons mené une étude détaillée des efforts magnétiques, notamment parasites, générés dans cette structure. Une méthode particulière a été développée pour représenter cette géométrie complexe et fondamentalement $3 D$.

ABSTRACT. This paper treats of an original motor-generator intended for electromechanical energy storage. It is a slotless synchronous machine with homopolar static field inductor. Its topology is discoid for better integration to the flywheel. Motor-generator don't have to disturb magnetic bearing of the flywheel set. We led a detailed study of magnetic forces, in particular, parasitical forces. Special method has been developped to represent this structure complex and interently $3 D$.

MOTS-CLES : moteur synchrone homopolaire, efforts parasites, efforts de Laplace, efforts réluctants, éléments finis, stockage électromécanique.

KEY WORDS: synchronous homopolar motor, parasitic forces, Laplace forces, reluctance forces, finite-elements, flywheel energy storage.

Revue Internationale de Génie Electrique Vol. 4 - n³-4/2001, pp.367-390. 


\section{Introduction}

Les énergies renouvelables représentent un énorme potentiel encore très peu exploité pour la production d'électricité, notamment [MUL 98]. Mais ces sources sont souvent fluctuantes et posent immédiatement le problème du stockage de l'électricité indispensable pour résoudre le problème de l'inadéquation production consommation. Dans les véhicules électriques, qu'ils soient tout électriques ou hybrides, le stockage de l'énergie constitue également un enjeu majeur.

On peut stocker l'énergie sous différentes formes: thermique, mécanique, chimique, magnétique, électrostatique ou biologique. Parmi tous les systèmes existants, le stockage électromécanique ou stockage inertiel se distingue par de bonnes possibilités en puissance massique et surtout par un nombre de cycles de fonctionnement très élevé permettant une très longue durée de vie [YON 96].

Pour stocker un maximum d'énergie il faut faire tourner une masse à très grande vitesse. La vitesse maximale est donnée par la limite de rupture du matériau, ce dernier doit par conséquent être à très haute limite de résistance mécanique [MAR 98].

Afin de minimiser les pertes, on utilise généralement des suspensions magnétiques ainsi qu'un vide partiel pour réduire les frottements aérodynamiques.

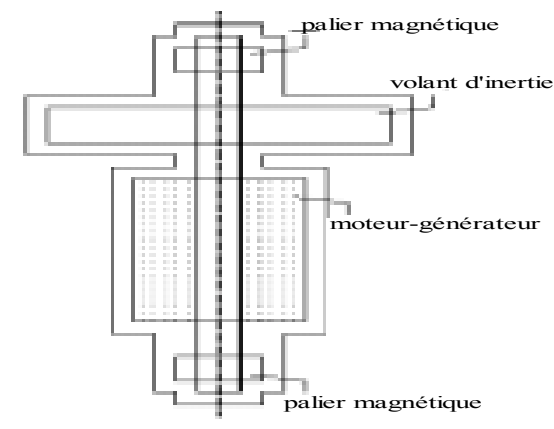

Figure 1. Système de stockage électrique inertiel dont les principaux composants sont dissociés

L'accumulateur étudié en collaboration avec des mécaniciens et le LEG ${ }^{1}$ [dans le cadre d'un plan pluri-formations] est destiné à la production décentralisée d'électricité par des sources photovoltaïques et/ou éoliennes où les contraintes (coût, durée de vie, temps de cycle) nous ont amené à choisir une architecture particulière intégrant un volant d'inertie cylindrique en acier [KER 99], un moteur-générateur et des suspensions magnétiques. Une structure discoïde particulièrement bien adaptée à cette application a été conçue et étudiée [BEN 99].

Dans ce qui suit nous présenterons le moteur-générateur développé par le LESiR $^{2}$. Puis, nous développerons la méthodologie utilisée pour l'évaluation des perturbations générées par le moteur-générateur dans un fonctionnement équilibré et lors d'un déséquilibre mécanique des disques rotoriques. Nous déterminerons les

\footnotetext{
${ }^{1}$ Laboratoire d'Electrotechnique de Grenoble

${ }^{2}$ Laboratoire d'Electricité Signaux et Robotique
} 
deux types d'efforts parasites générés par cette structure, dans un premier temps les efforts de Laplace puis les efforts réluctants.

Cette méthodologie sera ensuite appliquée à la maquette de validation. Les résultats obtenus seront enfin analysés.

\section{Le moteur-générateur du LESiR}

Nous avons cherché à réaliser un système volant / moteur-générateur / paliers le mieux intégré possible. Notre choix s'étant porté vers un volant cylindrique en acier haute résistance, nous avons recherché une structure de moteur-générateur s'intégrant au mieux au volant.

Les caractéristiques souhaitables d'un moteur-générateur pour le stockage électromécanique sont :

- Haute vitesse de rotation;

- Puissance maximale constante sue une plage de vitesse étendue (typiquement 1 à 3 );

- Haut rendement en charge ou en décharge ;

- Pertes d'auto-décharge aussi faibles que possible ;

- Bonne intégration au volant d'inertie cylindrique ;

- Faible coût.

Ces différentes contraintes nous imposent :

- Pour des raisons de résistance mécanique, rotor entièrement passif (ne comportant ni bobinage, ni aimant) et de préférence massif ;

- Pour minimiser les pertes magnétiques dans le fer, toutes les parties ferromagnétiques sont tournantes (fixes par rapport au champ magnétique);

- Bobinage induit fixe dans l'entrefer générant un champ tournant aussi parfait que possible ;

- Pour obtenir une large plage à puissance constante, une excitation bobinée réglable ;

- Pour atteindre un bon niveau d'intégration au volant d'inertie, une structure disque.

Un dispositif répondant simultanément aux contraintes précédemment mentionnées a été conçu dans l'équipe "conception d'actionneurs" à l'antenne de Bretagne du LESiR.

Ce dispositif est composé de deux disques dentés réalisés dans un matériau magnétique doux, un bobinage induit fixe triphasé situé dans l'entrefer et produisant une force magnétomotrice la plus sinusoïdale possible, et un bobinage inducteur solénoïdal fixe autour de l'axe des disques (voir figure 2). On notera que le rotor contribue au stockage d'énergie cinétique. Les dimensions sont fournies en annexe. 


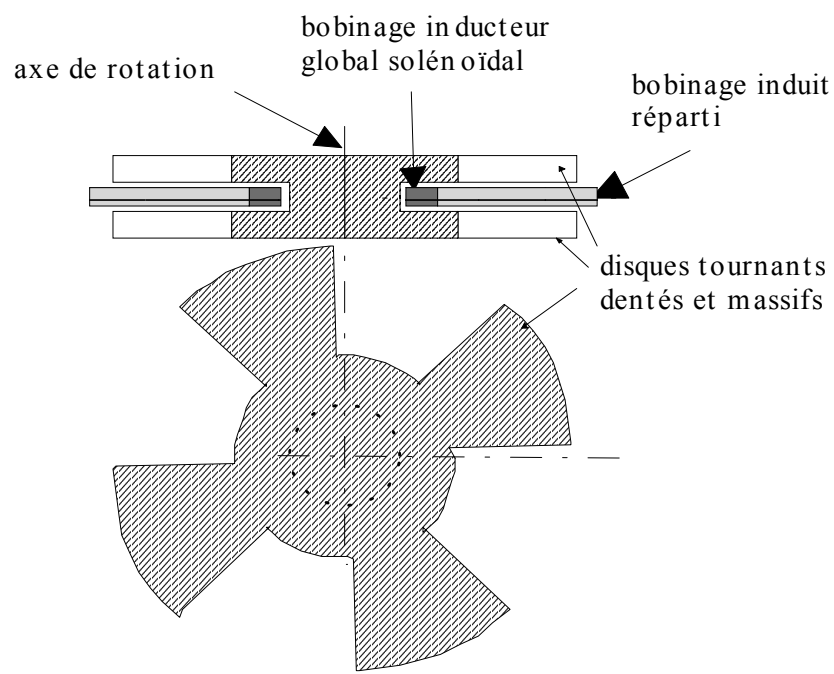

Figure 2. Moteur-générateur du LESiR

Le principe de fonctionnement est fondé sur la «commutation de flux » à savoir : l'alternance du flux inducteur vu par une phase du bobinage d'induit fixe est obtenue à partir d'une excitation par courant continu et par le déplacement du circuit réluctant pur.

Pour illustrer ce fonctionnement, prenons le cas d'une structure élémentaire monophasée représentative (voir figure 3) composée de dents magnétiques, d'encoches et de spires élémentaires. L'entrefer magnétique (jeu mécanique + hauteur spires) est magnétisé, à vide, par le bobinage d'excitation qui sur ce schéma est représenté par la d.d.p. magnétique générée aux bornes des deux rotors dentés.

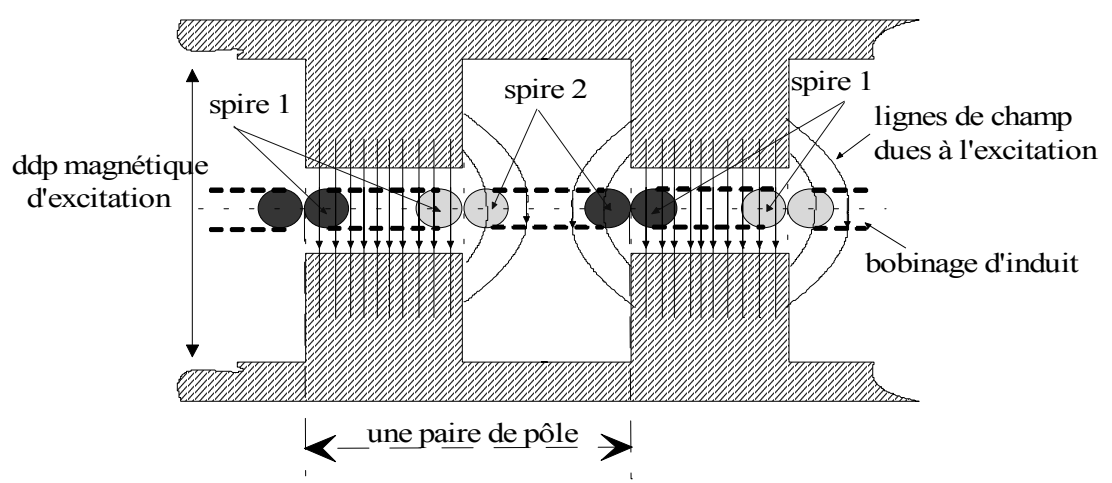

Figure 3. Représentation $2 D$ du principe

La variation du flux inducteur dans les spires 1 et 2 est représentée sur la figure 4. Ainsi, le flux inducteur total embrassé par les deux spires en séries formant le 
bobinage induit d'une phase de l'induit, correspond à la différence des deux flux, il est donc alternatif de composante moyenne nulle.

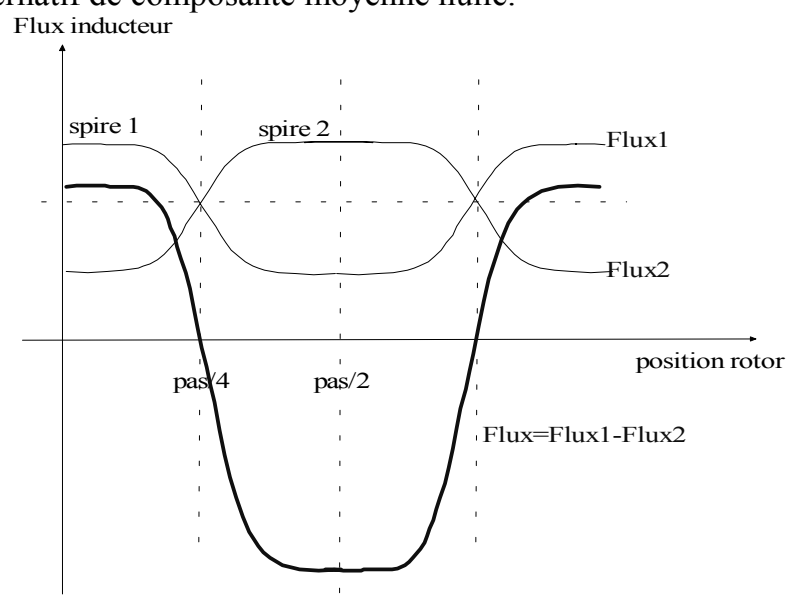

Figure 4. Flux inducteur embrassé par les deux spires élémentaires d'une phase de l'induit

L'injection, dans le bobinage induit, de courants synchronisés avec les f.e.m. permet d'obtenir un couple. Les forces magnétiques d'un tel dispositif à bobinage dans l'entrefer, sont essentiellement des forces de Laplace mais compte tenu de la réluctance variable des disques rotoriques, il existe des efforts réluctants. la valeur moyenne du couple réluctant est nulle du fait de la stratégie de commande utilisée (courants alternatifs de même période que les f.e.m.).

Afin de minimiser les fluctuations de couple ou de puissance et de réduire au minimum les pertes magnétiques dans les disques, le bobinage induit sera, de préférence, triphasé, alimenté par des courants sinusoïdaux et devra s'intégrer entre les deux disques. Pour ces raisons, après avoir réalisé une première maquette en bobinage filaire, nous avons utilisé une technologie sur circuit imprimé. Chaque phase du bobinage induit est composée de plaques de circuit imprimé double face appelée galette. L'induit, triphasé, est ainsi constitué de galettes superposées convenablement connectées.

Nous pouvons voir ci-contre (figure 5) une photographie d'une maquette que nous avons réalisée.

Figure 5. Maquette de validation réalisée au LESiR

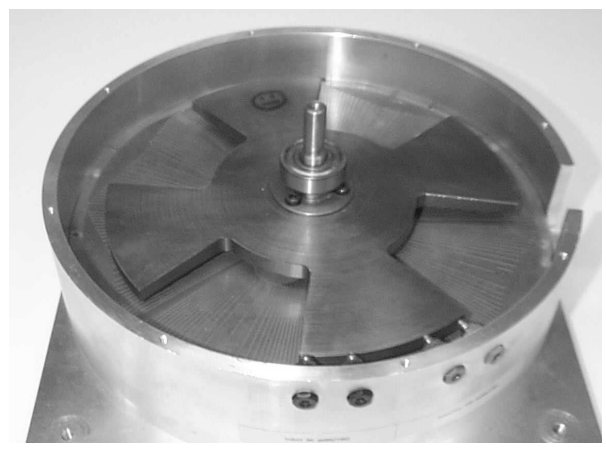


D'autre part afin de minimiser les pertes mécaniques, le système sera placé sous vide partiel et des paliers magnétiques assureront la sustentation de l'ensemble. Ces paliers magnétiques sont étudiés et réalisés au LEG [FAU 98].

Pour l'assemblage de ces deux systèmes et, en particulier, pour analyser les sollicitations des paliers magnétiques, il est nécessaire de quantifier les efforts parasites engendrés par le moteur générateur.

\section{Calcul des efforts parasites}

Pour une telle structure de moteur il existe deux types d'efforts, les efforts de Laplace générés par l'interaction du champ magnétique inducteur et les courants du bobinage induit $\mathrm{J}$, et les efforts réluctants dus à l'interaction du champ magnétique de l'induit et la réluctance des disques rotoriques. Nous allons faire l'étude de ces efforts lors de l'équilibre ou du déséquilibre du rotor provoqué par le déplacement des disques rotoriques par rapport au bobinage statorique. Nous avons déterminé six déplacements élémentaires possibles et de deux natures différentes :

- Les déplacements linéaires :
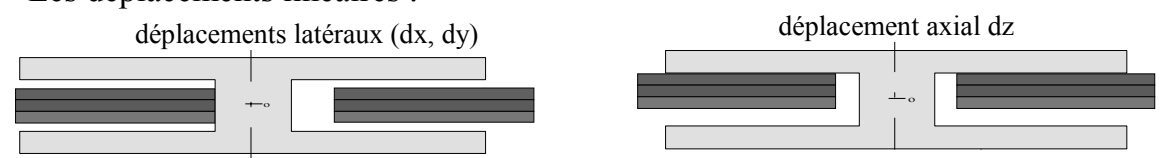

- Les déplacements angulaires :
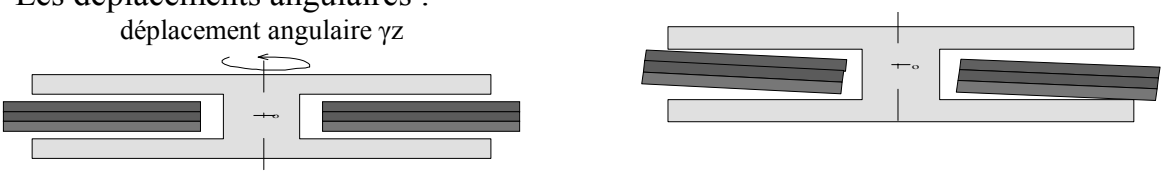

déplacements angulaires $(\gamma \mathrm{x}, \gamma \mathrm{y})$

Figure 6. Déplacements élémentaires possibles

Les résultats seront exprimés par la résultante des forces ainsi que le moment de cette résultante au point $\mathrm{O}$. Les moments autour des axes $\mathrm{x}, \mathrm{y}$ et $\mathrm{z}$ seront notés respectivement $\mathrm{Tx}$, Ty et Tz, Tz étant le couple moteur. De même les résultantes des forces dans les directions $\mathrm{x}$, y et $\mathrm{z}$ seront respectivement notées Fx, Fy et Fz.

\subsection{Efforts de Laplace}

Le circuit magnétique fonctionnant en régime linéaire et le bobinage induit étant dans l'air, l'évaluation de ces efforts peut donc s'effectuer par le modèle des forces de Laplace $\overrightarrow{d F}=\vec{J} \wedge \overrightarrow{B_{e}}$. Ainsi, la répartition de l'induction magnétique $B_{e}$ due à l'inducteur est déterminée à partir d'un calcul par éléments finis en 3D (flux3D 
[CED 98]), le bobinage induit étant absent. Quant à la répartition des courants d'induit $J$, elle sera exprimée à partir d'un modèle analytique en fonction d'un certain nombre de paramètres électriques et géométriques.

\subsubsection{Détermination de la répartition de l'induction due à l'inducteur}

Pour le calcul des efforts parasites, les calculs par éléments finis 3D ne sont utilisés que pour la détermination de la répartition dans l'espace inter-disques de l'induction due à l'inducteur. Cette répartition est ensuite exprimée sous forme de trois matrices inductions, radiale, orthoradiale et normale.

La structure totale est représentée sur la figure 7. Celle ci montre une symétrie axiale et azimutale, l'inducteur sous forme de solénoïde est représenté au centre.

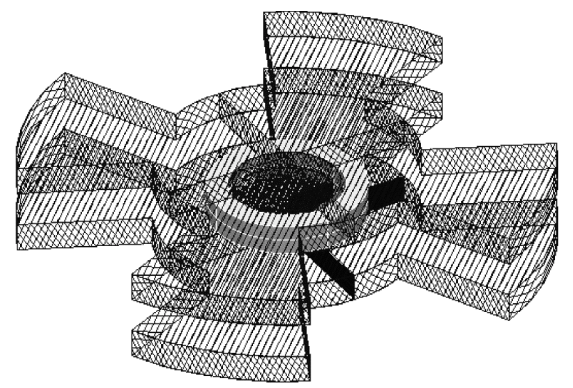

Figure 7. Structure complète du moteur avec l'inducteur

Compte tenu de ces symétries, ainsi que des possibilités du code de calcul utilisé, la structure exploitée est celle de la figure 8 .

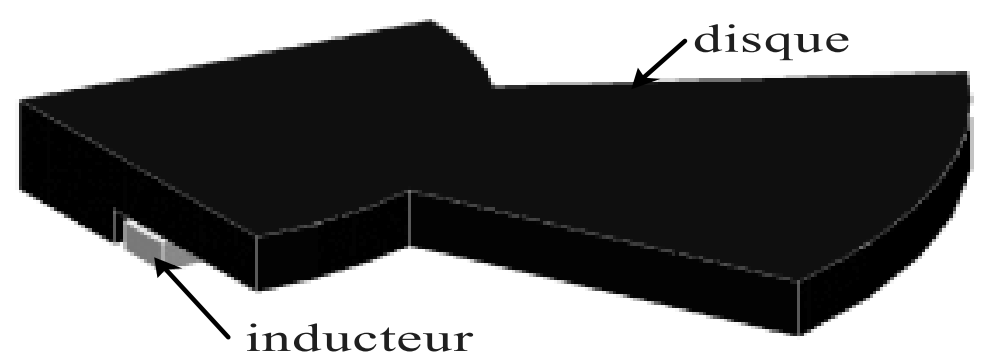

Figure 8. Motif élémentaire pour les calculs magnétiques $3 D$

Les calculs sont normalisés pour une densité de courant de $1 \mathrm{~A} / \mathrm{mm}^{2}$ dans l'inducteur et dans l'induit.

On obtient alors les courbes de l'induction dans l'entrefer des figures 9 et 10. Le premier réseau de courbes est donné pour des contours de rayon $\mathrm{r}=75 \mathrm{~mm}$ (rayon moyen de la dent) et situé à différentes hauteurs dans l'entrefer. Le deuxième réseau de courbe est donné pour des contours de hauteur $\mathrm{z}=0 \mathrm{~mm}$ (milieu de l'entrefer) et pour différents rayons. 


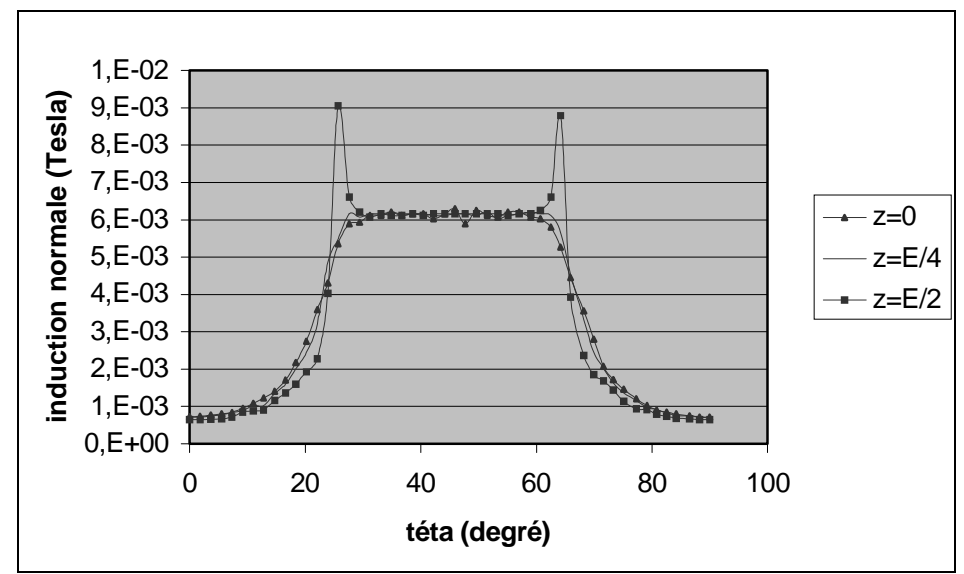

Figure 9. Induction normale sous le disque au rayon moyen $r=75 \mathrm{~mm}$

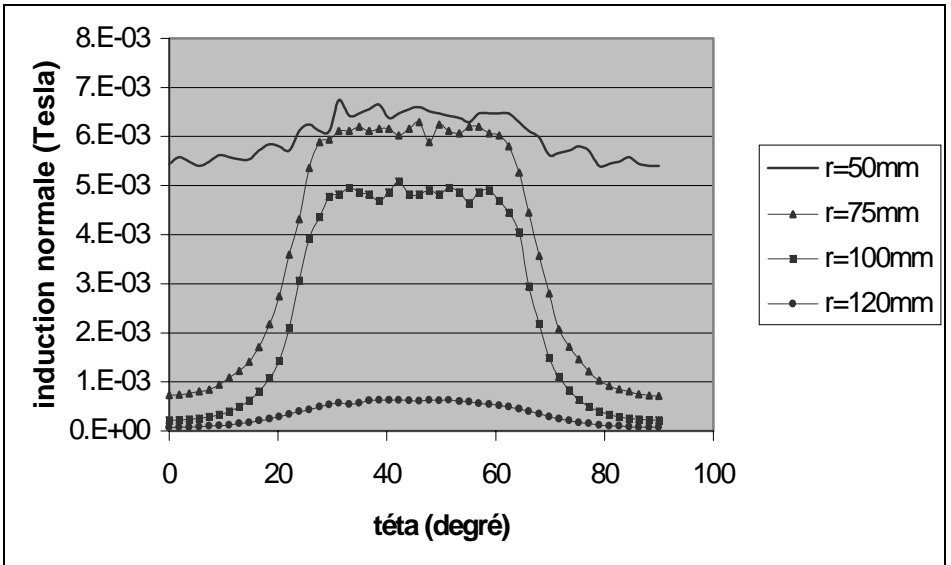

Figure 10. Induction normale au milieu de l'entrefer $z=0 \mathrm{~mm}$

On remarque sur la figure 9 que pour un rayon donné l'induction sous la dent $\left(25^{\circ} \leq \theta \leq 65^{\circ}\right)$ est constante excepté pour la courbe $\mathrm{z}=\mathrm{E} / 2$, on se trouve alors juste en dessous du disque et l'on observe un effet de pointe dû à la forme de la dent.

Quant à la figure 10, elle nous permet d'observer le flux de "fuite" sachant que le rayon de fond d'encoche est $R_{\text {int }}=50 \mathrm{~mm}$ et le rayon extérieur d'une dent est $R_{e x t}=100 \mathrm{~mm}$. Une autre illustration de ces « fuites » est donnée à la figure 11. 


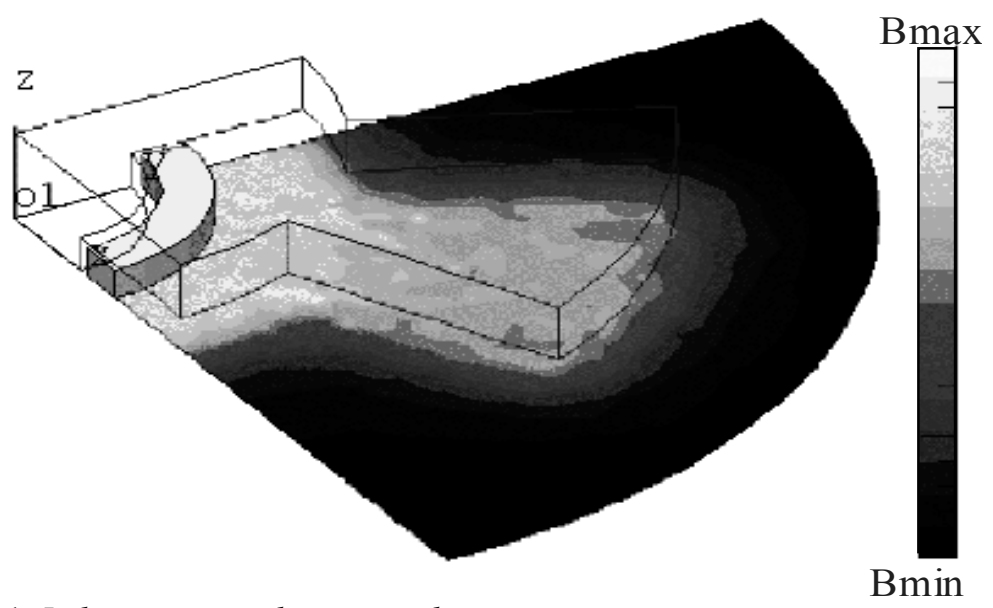

Figure 11. Induction normale sous un disque

Nous avons jusqu'à présent observé les inductions normales dans l'entrefer génératrices du couple moteur. Or les composantes de l'induction radiales et orthoradiales sont essentiellement à l'origine des efforts parasites. Ces dernières sont dues au flux de fuite sortant des bords des dents (fond d'encoche, extrémités des dents et côtés des dents). Nous pouvons en avoir une image qualitative sur les figures ci-dessous. Ces dernières montrent notamment que la composante radiale de l'induction agira essentiellement sur les têtes de bobines.

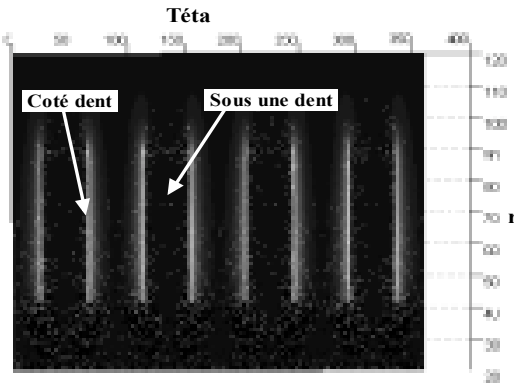

Figure 12. Induction orthoradiale Bmin dans l'entrefer

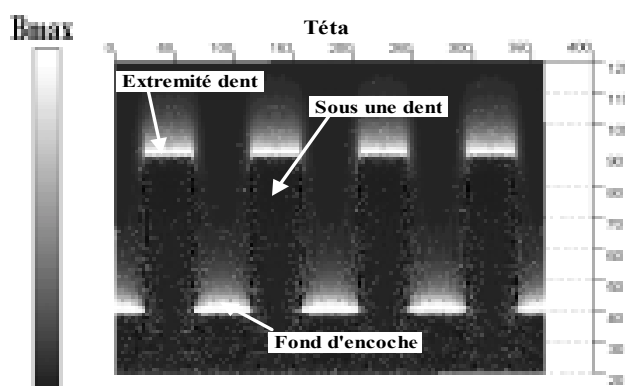

Figure 13. Induction radiale dans l'entrefer

\subsubsection{Modélisation de l'induit}

L'induit actuel est constitué de galettes (circuit imprimé double face), il y en a deux par phase. Les pistes sont dimensionnées de manière à obtenir une force magnétomotrice sinusoïdale. Ainsi, ceci a nécessité la conception de pistes dont la largeur est variable (Figure 14). Lorsque nous parlerons de la densité de courant dans l'induit, il s'agira de la densité de courant dans les pistes de sections minimales, soit la densité de courant maximale. 

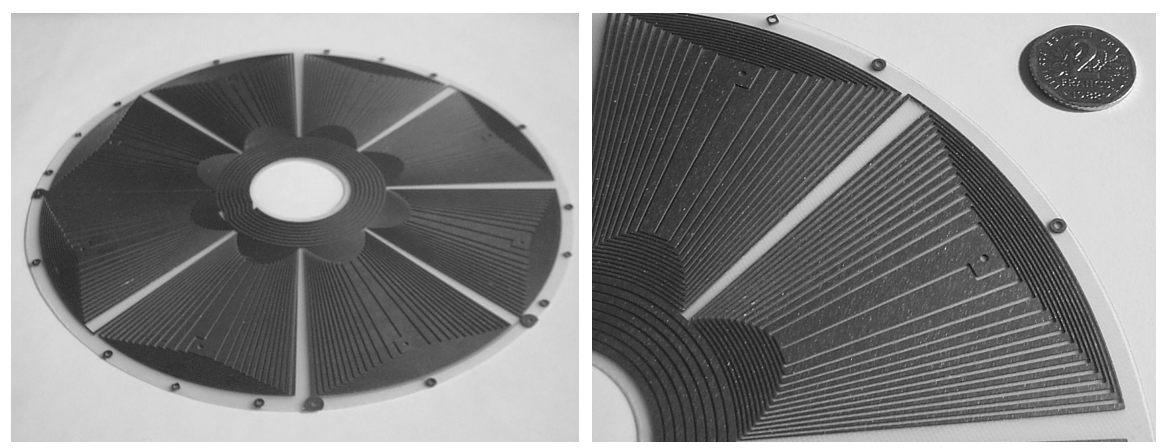

Figure 14. Bobinage induit du moteur générateur réalisé sur technologie circuit imprimé double face

Le modèle analytique du bobinage induit consiste en l'écriture des matrices des densités de courants aux même points que pour les matrices inductions, c'est à dire sur l'ensemble des points que nous définirons par leurs coordonnées $\left(M_{r}, M_{\theta}, M_{z}\right)$. Avec $-M_{r}$ : Ensemble des coordonnées radiales de tous les points.

$-M_{\theta}$ : idem pour les coordonnées angulaires. axiales.

$-M_{z}:$ idem pour les coordonnées

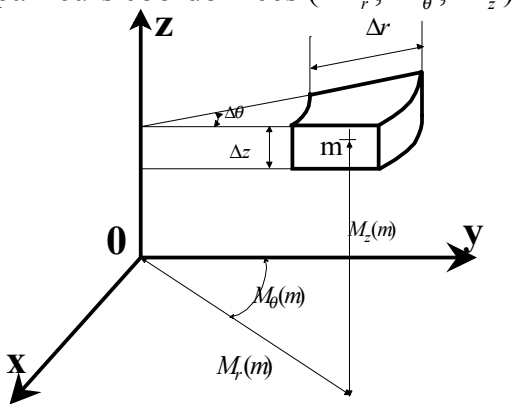

Figure 15. Elément de volume

Pour simplifier la modélisation, nous considérons le bobinage induit comme étant un volume de cuivre dans lequel la répartition de la densité de courant est pondérée par une fonction de « remplissage » notée $k_{r}$.

Le coefficient global de remplissage en cuivre est défini par $k_{r}=k_{r s} \cdot k_{r z}$ avec $k_{r s}$ le coefficient de remplissage surfacique qui tient compte de l'isolant entre deux pistes et $k_{r z}$ le coefficient de remplissage qui tient compte de la présence de la plaque d'époxy entre deux plaques de cuivres.

D'autre part chaque pôle est décomposé en quatre zones dans lesquelles la densité de courant est simplement radiale ou azimutale (voir figure 16). Un changement de polarité s'obtient par une simple inversion de signe. 


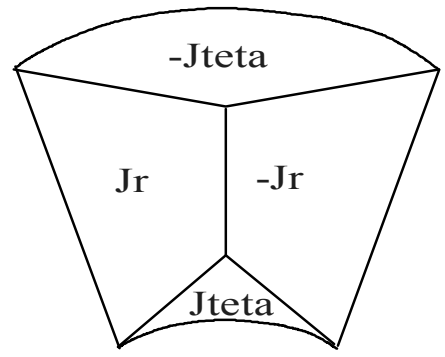

Figure 16 . Définition des zones d'un pôle d'induit

A partir de ces fonctions et de ces coefficients, un programme a été réalisé sous environnement Matlab, qui donne pour un point quelconque dans le volume d'entrefer la densité de courant. On obtient alors, à un instant donné, les densités de courants radiale et orthoradiale, représentées ci-dessous, la composante normale $J_{z}$ étant nulle.

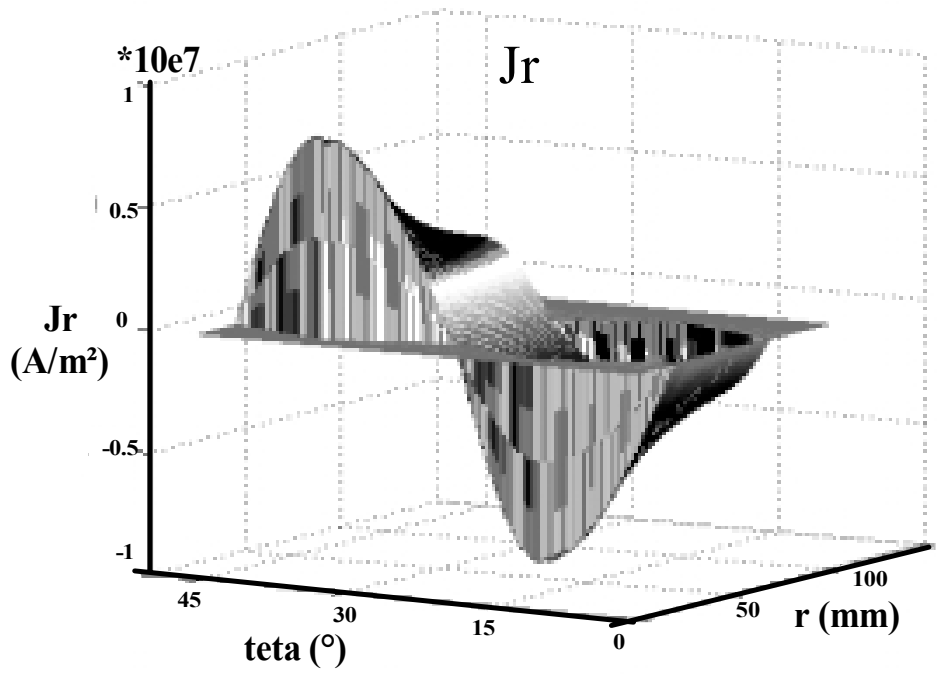

Figure 17. Densité de courant radiale 


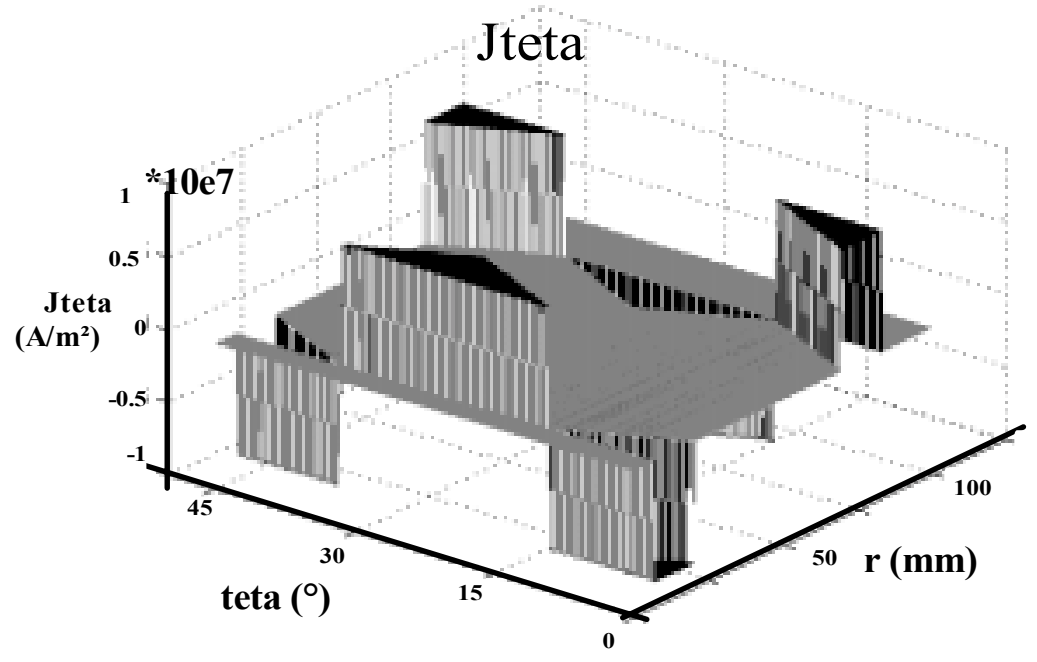

Figure 18. Densité de courant orthoradiale

\subsubsection{Principe de calcul}

L'évaluation des efforts parasites a été effectuée dans le cas de la maquette de validation pour une densité de courant dans l'inducteur de $1 \mathrm{~A} / \mathrm{mm}^{2}$ et pour une densité de courant maximale dans l'induit également de $1 \mathrm{~A} / \mathrm{mm}^{2}$ En régime linéaire les efforts seront proportionnels au produit des densités de courants. L'étude fournit donc en quelque sorte des valeurs normalisées.

Nous rappelons que le bobinage induit est triphasé et composé de deux galettes par phase. Compte tenu de la répartition de l'induction dans l'espace inter disque, la position relative des galettes influe sur le fonctionnement du moteur-générateur. Nous définissons deux répartitions des phases différentes, la configuration symétrique (figure 19) et la configuration asymétrique (figure 20).

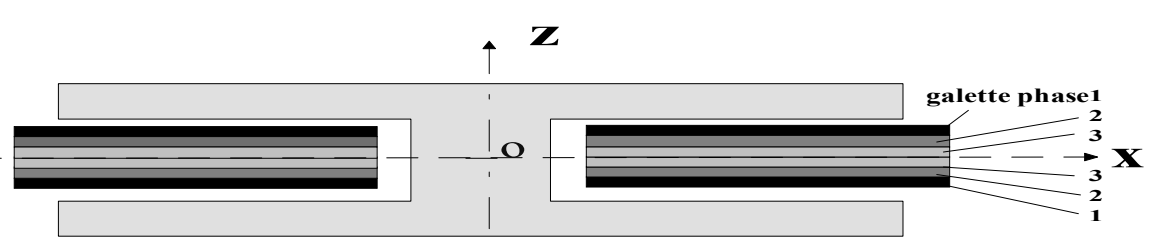

Figure 19. Configuration symétrique 


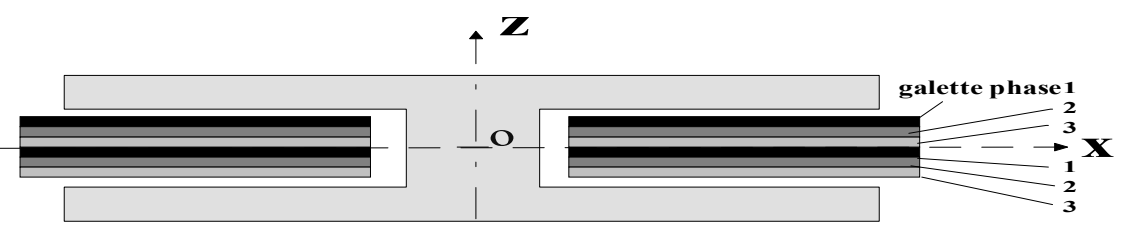

Figure 20. Configuration asymétrique

Etant donné les symétries de la structure de l'induit et du champ inducteur, on peut remarquer que la configuration «symétrique » va minimiser les perturbations. En effet les perturbations créées localement s'éliminent entre elles au niveau global. En effet ces dernières sont dues à l'interaction du courant induit et des composantes radiales et orthoradiales de l'induction, et, avec cette configuration le courant présente une symétrie par rapport au plan horizontal Oxy, alors que les composantes radiales et orthoradiales de l'induction présentent elles une anti-symétrie par rapport à ce même plan.

Cependant cette répartition des phases va engendrer un déséquilibre des f.e.m. à cause, notamment, de l'effet de pointe (figure 9) vu essentiellement par la phase 1. C'est en disposant les phases d'une manière successives (configuration " asymétrique") qu'on réussira à minimiser le déséquilibre des f.e.m. dû à l'effet de pointe. Ainsi la configuration asymétrique sera plus favorable en terme d'ondulation de couple.

Nous disposons de l'induction et de la densité de courant en chaque point, on peut alors calculer l'effort volumique $f_{v}$ ainsi que le moment volumique $m_{v}$ en chaque point. On obtient alors trois matrices d'efforts volumiques et trois matrices de moments volumiques.

De tous ces éléments volumiques on peut, en intégrant sur tout le volume des galettes, déterminer l'effort résultant $\mathrm{F}$ appliquée au point $\mathrm{O}$, centre du circuit magnétique :

$$
F=\sum_{\text {volume_galettes }} f_{v} \cdot 2 \pi \cdot r \cdot \Delta r \cdot \Delta z \cdot \Delta \theta
$$

Pour une discrètisation uniforme, cette expression devient :

$$
F=2 \pi \cdot \Delta r \cdot \Delta z \cdot \Delta \theta \cdot \sum_{\text {volume_galettes }} r \cdot f_{v}
$$

De même pour le moment :

$$
T=2 \pi \cdot \Delta r \cdot \Delta z \cdot \Delta \theta \cdot \sum_{\text {volume_galettes }} r \cdot m_{v}
$$

Ainsi nous pouvons résumer la méthodologie que nous avons employée par l'organigramme ci dessous. 
14 Revue Internationale de Génie Electrique.

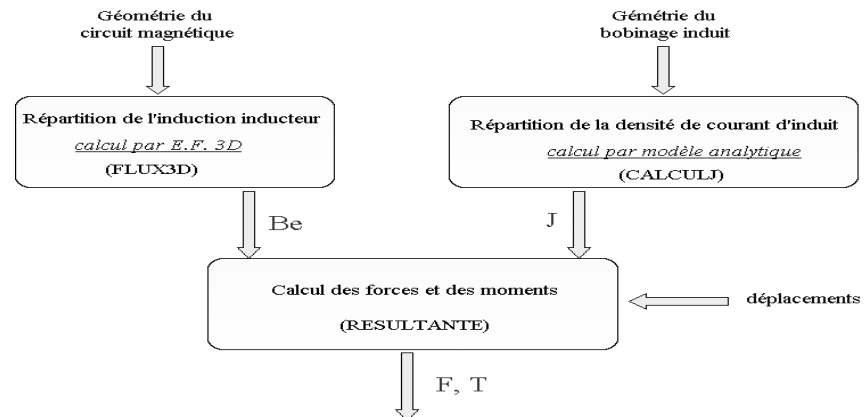

Figure 21. Méthode de calcul des efforts de Laplace

\subsubsection{Résultats et analyses des effets sur le couple moteur}

On voit que pour une rotation $\gamma \mathbf{z}$, le couple moteur pour une seule galette est quasiment sinusoïdal (figure 22) avec une valeur moyenne non nulle. La somme du couple des six galettes nous donne le couple total. On observe que celui ci présente une ondulation de pulsation $2 \omega$ due essentiellement à l'harmonique 3 de l'induction généré par l'inducteur, les harmoniques de rang paire correspondants s'éliminant par le bobinage induit. On note d'autre part que la configuration asymétrique (courbe en en trait interrompus) donne une ondulation de couple moins importante.

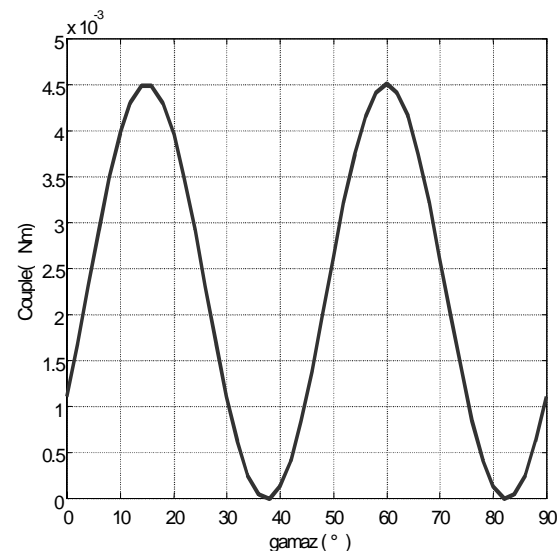

Figure 22. Couple d'une galette fonction d'une rotation $\gamma z$

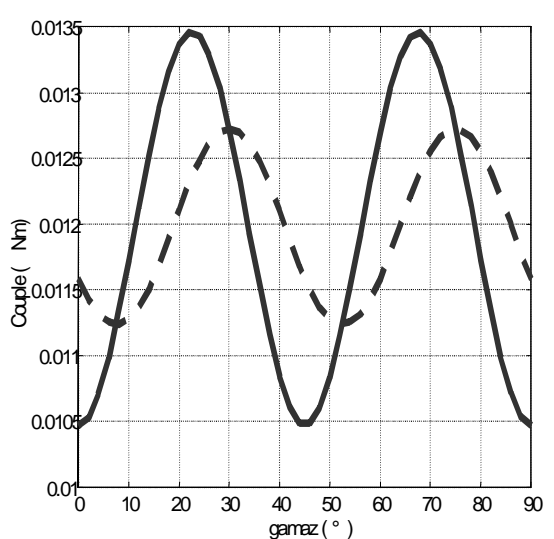

en Figure 23. Couple total en fonction d'une rotation $\gamma z$ 
Pour un déplacement latéral $\mathbf{d x}$, la partie active de l'induit se trouvant sous le champ inducteur étant diminuée, il provoque donc la même diminution du couple moteur pour les deux configurations

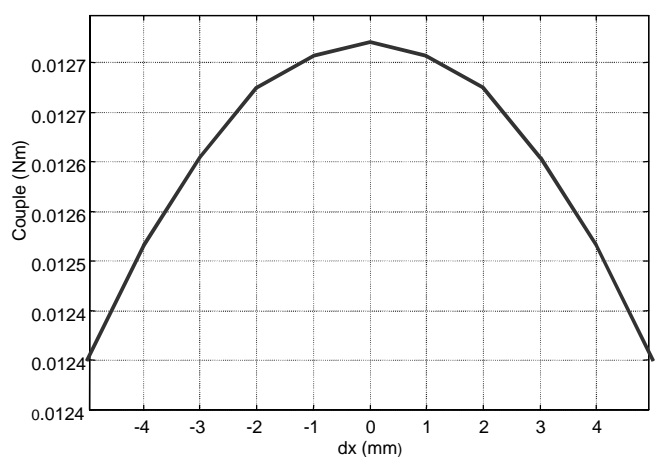

Figure 24. Couple total en fonction d'un déplacement $d x$

Pour un déplacement axial dz, limité par le jeu mécanique $(d z= \pm 2 \mathrm{~mm})$ on observe une augmentation du couple pour les deux configurations du fait de l'effet de pointe de l'induction due à l'inducteur signalé précédemment, le bobinage s'approchant du disque.

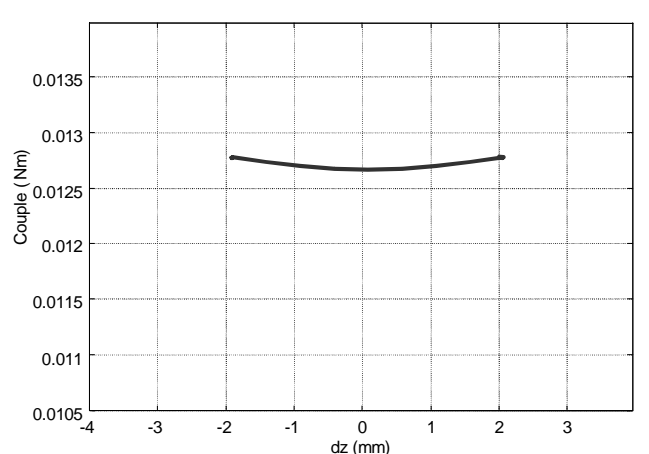

Figure 25. Couple total en fonction d'un déplacement $d z$

\subsubsection{Résultats et analyses des effets sur les efforts parasites}

- Effets d'une rotation $\gamma z$ :

Outre l'effet sur le couple moteur, cette rotation à également un effet sur la composante en $\mathrm{z}$ de la résultante des forces (Figure 21), celle qui tend à lever la galette..Celle ci est due à la composante orthoradiale de la densité de courant $J_{\theta}$ et à l'induction radiale $\mathrm{Br}$. On voit sur la figure 27 une illustration de la cause de cette force.

On remarque au passage que la configuration symétrique (courbe continue) rend nul cet effort lorsqu'il n'y a aucun déplacement. Alors que pour la configuration asymétrique le module de la force axiale varie sinusoïdalement en fonction de la rotation des disques. 


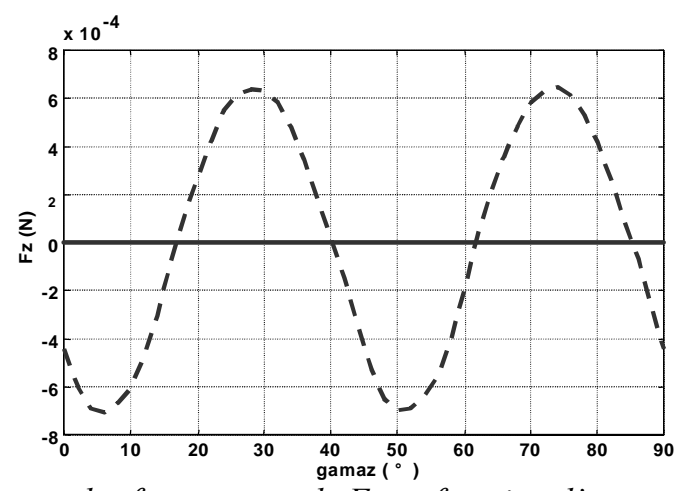

Figure 26. Résultante des forces normale Fz en fonction d'une rotation $\gamma z$

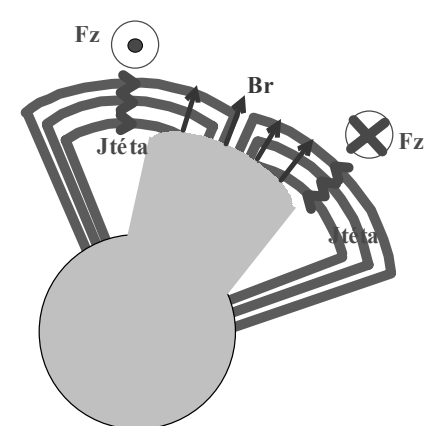

Yz tel que $R z=0 N$

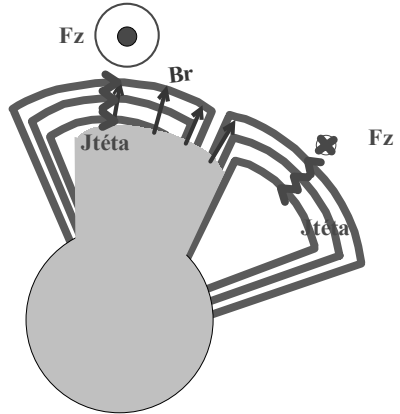

Yz tel que $R z \approx R z_{\text {max }}$

Figure 27. Illustration des causes de la présence d'une résultante des forces axiale $F z$ lors d'une rotation $\gamma \mathrm{z}$

- Effets d'un déplacement axial dz:

Les effets de ce déplacement ont été calculés pour une rotation $\gamma \mathrm{z}=30^{\circ}$ car le couple moteur a la même valeur pour les deux configurations (symétrique et asymétrique). Ce déplacement n'a d'influence que sur le couple moteur et sur la résultante des forces axiale Fz. On voit sur la figure 28 que ce déplacement crée une résultante des forces $\mathrm{Fz}$ qui tend à déplacer les galettes dans le même sens que le déplacement, il a donc un effet déstabilisant pour le système. La composante normale de la résultante des forces $\mathrm{Fz}$ étant due à l'induction radiale $\mathrm{Br}$ et vu que plus on s'approche du disque plus l'induction radiale $\mathrm{Br}$ est grande, alors la résultante des forces $\mathrm{Fz}$ augmente. 


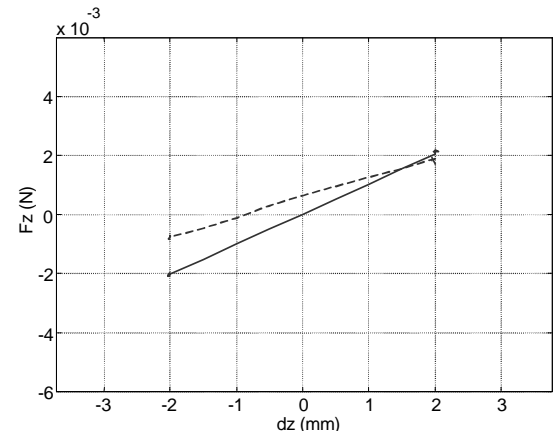

Figure 28. Résultante des forces axiale Fz en fonction d'un déplacement $d z$

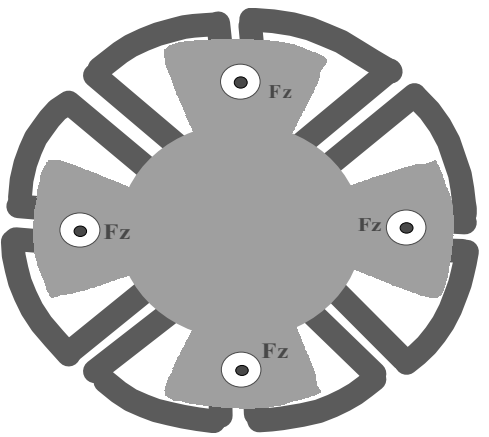

Figure 29. vue de dessus du système lors d'un déplacement $d z$

La figure 30 illustre ce déplacement. On remarque que pour les dents 1 et 3 on a exactement le même effort axiale Fz. Il ne peut donc pas y avoir de moment Tx et de résultante des forces axial Fy. Mais ce n'est pas le cas pour les dents 2 et 4 . On a interaction entre la densité de courant azimutale $J_{\theta}$ et l'induction normale $\mathrm{Bz}$ d'où la création d'une résultante des forces axiale Fx. De plus les têtes de bobines en face de ces dents ne voient pas la même valeur de l'induction radiale $\mathrm{Br}$ donc les efforts normaux Fz sur les dents 2 et 4 sont différents d'où la création d'un moment Ty.

De plus, comme nous l'avions signalé précédemment la partie active du courant d'induit sous la dent 4 voit une induction normale $\mathrm{Bz}$ plus faible, donc il y a une diminution du couple moteur.

On notera également l'effet stabilisant de la résultant des forces Fx dans le cas de la configuration asymétrique. Cette dernière est quasi nulle dans le cas de la configuration symétrique.

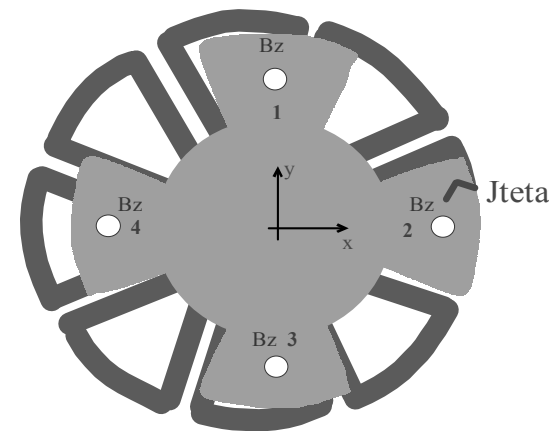

Figure 30. vue de dessus du système lors d'un déplacement $d x$ 

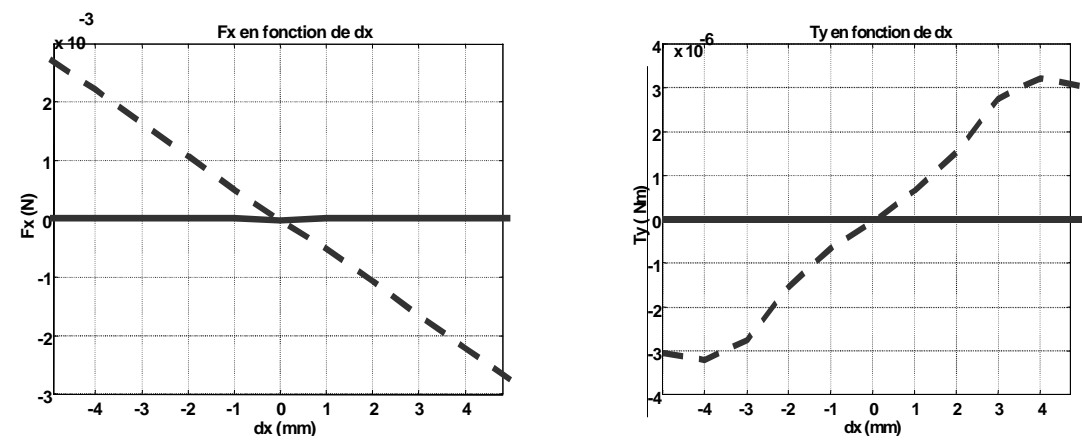

Figure 31. Effort parasites lors d'un déplacement $d x$

\subsubsection{Conclusion sur les efforts de Laplace}

Dans cette partie nous avons décrit la méthodologie de calculs des forces et donné les résultats obtenus sur les efforts et moments de Laplace qui s'exercent sur la structure particulière étudiée. Ces résultats ont ainsi montré que pour une densité de courant dans l'inducteur de $10 \mathrm{~A} / \mathrm{mm}^{<}$, et une densité de courant maximale dans l'induit de $10 \mathrm{~A} / \mathrm{mm}^{<}$, on a

- un effort radial Fx, Fy de l'ordre du Newton.

- un effort axial Fz de l'ordre du dixième de Newton.

- un moment radial Tx,Ty de l'ordre du milli-Newton-mètre.

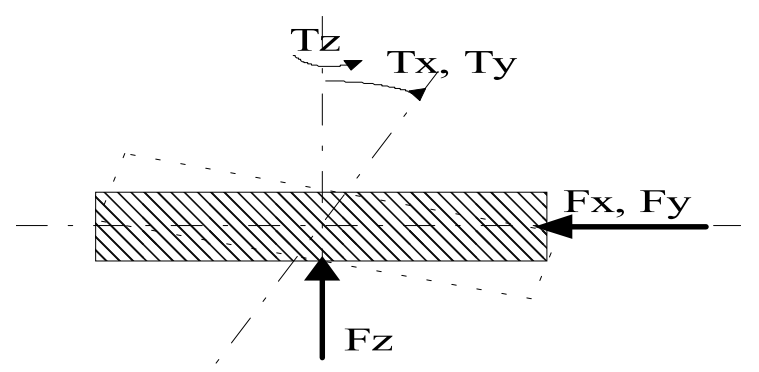

Figure 32. Résultantes des forces et moments sollicitant les paliers

De plus, la disposition relative des galettes des trois phases influe aussi sur le couple moteur et sur les efforts parasites. Nous avons ainsi montré que la configuration symétrique, à l'inverse de la configuration asymétrique, permet de minimiser (voire annuler) les efforts parasites au détriment d'une ondulation de couple plus importante. Cependant compte tenu des faibles niveaux de perturbations, qui pourront être compensées par les paliers magnétiques, nous avons intérêt pour limiter 1 'ondulation du couple à choisir la configuration asymétrique pour laquelle le taux d'ondulation du couple moteur est d'environ $6 \%$. 


\subsection{Efforts réluctants}

Jusque là nous n'avons considéré que les forces de Laplace, nous devons compléter cette analyse en étudiant les efforts réluctants dus à l'interaction du rotor (ferromagnétique) avec le champ magnétique généré par l'induit (le courant inducteur étant nul).

\subsubsection{Calcul des efforts réluctants}

D'après la structure du système, le bobinage induit se trouvant entre deux disques ferromagnétiques, on sait qu'un déplacement $\mathrm{dz}$ aura un effet déstabilisant pour le système, le bobinage induit s'approchant de l'un des deux disques. Pour quantifier cet effort il faudrait modéliser l'induit sous flux3D par une répartition de densité de courant volumique comme celle décrite sur les figures 17 et 18 . Mais la version dont nous disposons ne nous a pas permis un tel calcul. Ainsi nous avons utilisé un modèle 2D de ce moteur-générateur et un logiciel de calcul par éléments finis en 2D. Le modèle que nous avons utilisé est celui décrit ci dessous, il a été établi d'après le parcours moyen d'une ligne de champ pour un quart du moteur générateur.

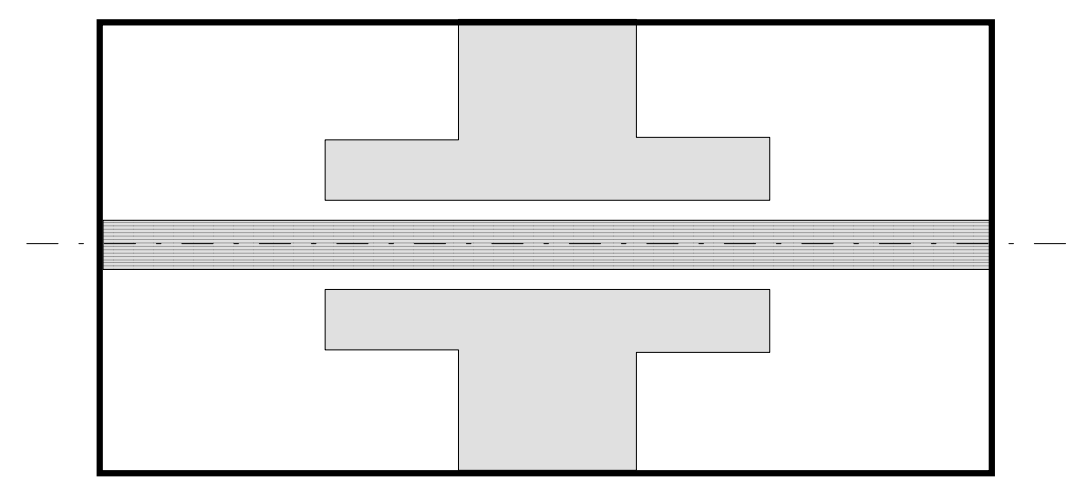

Figure 33. modèle $2 D$ du moteur-générateur

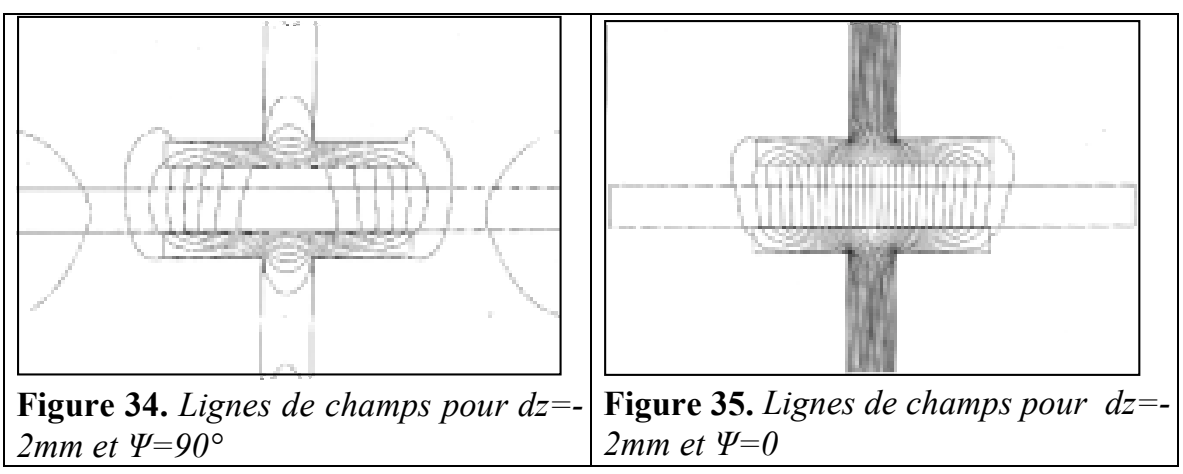


Nous avons réalisé ce calcul pour un déplacement $\mathrm{dz}$ variant de $-2 \mathrm{~mm}$ à $2 \mathrm{~mm}$, pour un angle d'auto pilotage $\Psi$ variant de $0^{\circ}$ à $90^{\circ}$ et pour une densité de courant maximale $J_{\text {induit }} \max =1 \mathrm{~A} / \mathrm{mm}^{2}$.

Le logiciel de calcul par éléments finis en 2D que nous utilisons, nous permet de faire le calcul des efforts sur ce motif. Comme pour le calcul des efforts électromagnétiques il faut rajouter un coefficient correcteur tenant compte du rapport des surfaces du calcul $2 \mathrm{D}$ et de la surface réelle ainsi que du coefficient de remplissage. Les résultats obtenus pour une densité de courant maximale $J_{\text {induit }} \max =1 \mathrm{~A} / \mathrm{mm}^{2}$ sont alors les suivants.

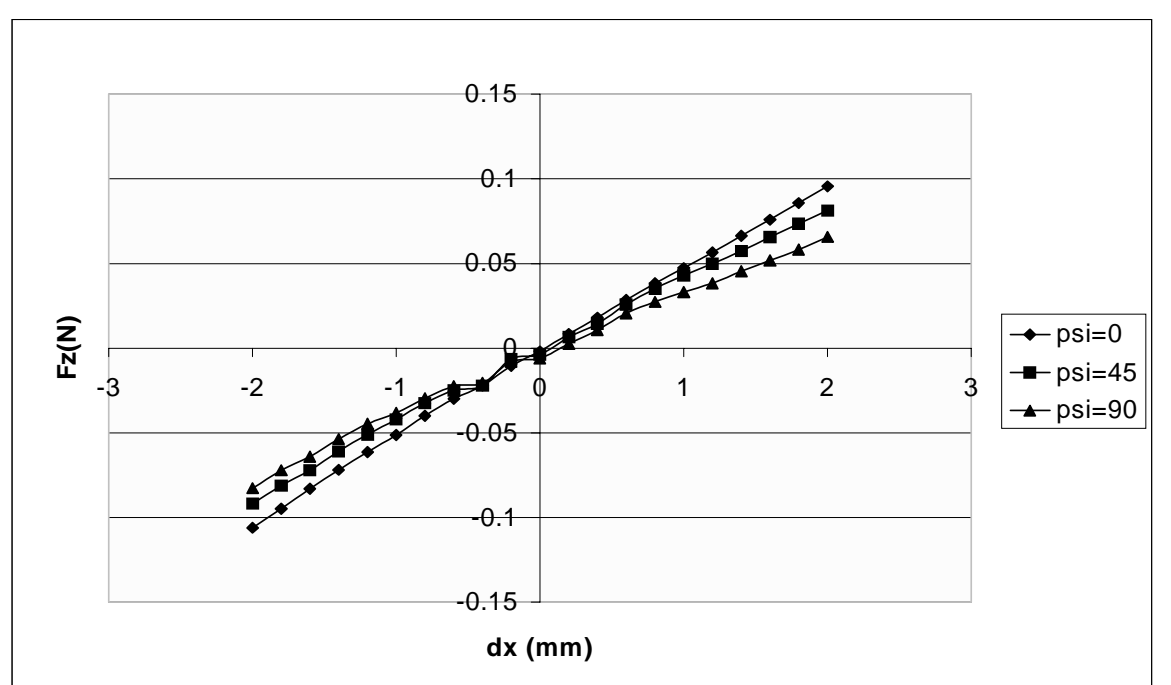

Figure 36. Composante normale de l'effort réluctant $F z$ en fonction de $d z$ paramètré en $\Psi$

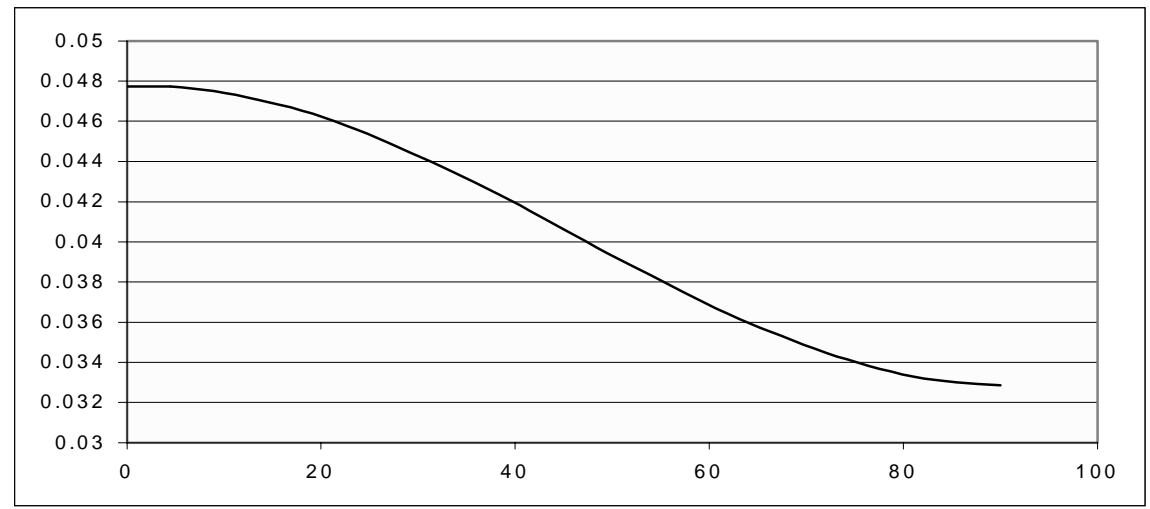

Figure 37. Effort réluctant normal Fz en fonction de l'angle d'autopilotage $\Psi$ pour $d z=1 \mathrm{~mm}$ 
Nous pouvons également conclure qu'un déplacement latéral $(\mathrm{dx}, \mathrm{dy})$ générera un effort (Fx, Fy) stabilisant afin de maximiser les surfaces de passage du flux d'induit. De même nous savons qu'un déplacement angulaire $\gamma \mathrm{x}$ générera un moment Ty instable pour le système.

\subsubsection{Conclusion sur les efforts réluctants}

Dans cette partie nous avons calculé les efforts réluctants développés par ce moteur-générateur. Cependant à cause de la limite de nos outils de calculs, nous n'avons pu calculer que l'effort Fz lors d'un déplacement dz. Ainsi l'effort axial généré par un déplacement $\mathrm{dz}$ de $1 \mathrm{~mm}$ pour une densité de courant maximale dans l'induit de $10 \mathrm{~A} / \mathrm{mm}^{<}$, est de l'ordre de $5 \mathrm{~N}$.

De plus, on a noté que l'influence de l'angle d'autopilotage est à variation sinusoïdale mais n'influe pas sur la nature de l'effort.

\section{Conclusion}

Ce travail avait pour but d'évaluer les efforts parasites générés par un moteurgénérateur d'un nouveau type associé à une suspension magnétique, pour une application de stockage électromécanique d'énergie.

Un calcul par éléments finis 3D nous a permis tout d'abord de déterminer la répartition de l'induction, dans l'entrefer, due à l'inducteur. L'interaction de cette dernière avec le bobinage induit, modélisé par une répartition volumique de densité de courant, a permis de calculer les efforts parasites qui sollicitent la suspension magnétique en fonctionnement équilibré et lors d'un déséquilibre.

Puis un calcul par éléments finis 2D nous a permis de quantifier les efforts réluctants.

Ainsi, appliqué à la maquette de validation, les calculs ont montré que pour un fonctionnement nominal $\mathrm{du}$ moteur les paliers magnétiques doivent être dimensionnés pour supporter une raideur radiale et axiale de l'ordre du Newton par millimètre et une sollicitation à un moment radial de l'ordre du milli-Newton-mètre pour les efforts de Laplace et une raideur axiale de $5 \mathrm{~N} / \mathrm{mm}$ pour les efforts réluctants.

Nous avons aussi montré que la configuration symétrique du bobinage induit, à l'inverse de la configuration asymétrique, permet de minimiser (voir annuler) les efforts parasites au détriment d'une ondulation de couple plus importante. Cependant compte tenu des faibles niveaux de perturbations nous avons intérêt à choisir la configuration asymétrique. 
22 Revue Internationale de Génie Electrique.

Ainsi nous pouvons résumer les performances des paliers dans le tableau suivant :

\begin{tabular}{|c|c|c|c|c|}
\hline Raideurs & $\mathrm{dx}$ & $\mathrm{dz}$ & $\gamma \mathrm{z}$ & $\gamma \mathrm{y}$ \\
\hline $\begin{array}{c}\text { Efforts } \\
\text { réluctants }\end{array}$ & Stable & $\begin{array}{c}\text { Instable } \\
\mathrm{dFz}=5 \mathrm{~N} / \mathrm{mm}\end{array}$ & Néant & Instable \\
\hline $\begin{array}{c}\text { Efforts de } \\
\text { Laplace }\end{array}$ & $\begin{array}{c}\text { Stable } \\
\mathrm{dFx}=0.05 \mathrm{~N} / \mathrm{mm}\end{array}$ & $\begin{array}{c}\text { Instable } \\
\mathrm{dFz}=0.1 \mathrm{~N} / \mathrm{mm}\end{array}$ & $\begin{array}{c}\text { Fz varie } \\
\text { sinusoïdalement } \\
\mathrm{dMx}=1 e^{-4} \mathrm{Nm} / \mathrm{mm}\end{array}$ & Néant \\
\hline
\end{tabular}

6. Bibliographie

[BEN 99] H. BEN AHMED, B. MULTON, J. DELAMARE, C. KERZRÉHO, J.Y.

COGNARD, Moteur/générateur discoïde à réluctance excitée et à bobinage dans l'entrefer, Brevet Français Nº9 15494 déposé le 8 décembre 1999, déposant : CNRS.

[CED 98] Notice d'utilisation de Flux3D version 220H, CEDRAT SA janvier 1998.

[FAU 98] F. FAURE, Etude d'un palier magnétique à un axe actif, DEA de Génie Electrique du Collège doctoral de l'INPG 1998.

[KER 99] C. KERZREHO, J.Y. COGNARD, G. DUMONT, Définition et optimisation de la structure d'une batterie électromécanique intégrée, 14ème Congrès Français de Mécanique, Toulouse, septembre 1999.

[MAR 98] A. MARQUET, C. LEVILLAIN, A. DAVRIU, S. LAURENT, P. JAUD, Stockage d'électricité dans les systèmes électriques, Technique de l'ingénieur D4030 5- 1998.

[MUL 98] B. MULTON, L'énergie sur la terre: analyse des ressources et de la consommation. La place de l'énergie électrique, revue 3EI septembre 1998.

[YON 96] J.P YONNET., Le stockage d'énergie par volant d'inertie, journéee SEE 1 février 1996 . 
Annexe :

\begin{tabular}{|l||c||c||}
\hline Caractéristiques & & Valeurs \\
\hline \hline Rayon extérieur & $R_{e x t}$ & $100 \mathrm{~mm}$ \\
\hline \hline Rayon de fond d'encoche & $R_{\text {int }}$ & $50 \mathrm{~mm}$ \\
\hline \hline Epaisseur des disques & $E_{d i s}$ & $10 \mathrm{~mm}$ \\
\hline \hline Jeu mécanique & $E$ & $1 \mathrm{~mm}$ \\
\hline \hline Hauteur du bobinage induit et inducteur & $H_{b}$ & $10.5 \mathrm{~mm}$ \\
\hline \hline Arc dentaire & $\tau_{d}$ & $40^{\circ}$ \\
\hline \hline Nombre de spires de l'inducteur & $N_{e}$ & 96 \\
\hline \hline Nombre de spires de l'induit & $N_{i}$ & 156 \\
\hline \hline Perméabilité du matériau des disques & $\mu_{r}$ & 1000 \\
\hline \hline
\end{tabular}

Olivier GERGAUD est agrégé de Génie Electrique (1998), ancien élève de l'Ecole Normale Supérieure de Cachan. Son stage de DEA (INP Grenoble 1999) concernait l'étude développée dans cet article. Il commence, à l'antenne de Bretagne du LESiR, une thèse de doctorat de l'ENS de Cachan sur l'Optimisation énergétique d'un système de production d'électricité d'origine renouvelable couplé au réseau et associé à un dispositif de stockage d'énergie.

Hamid BEN AHMED est maître de conférences à l'Antenne de Bretagne de l'ENS Cachan (département Mécatronique) et chercheur au LÉSiR (UPRESA CNRS). Ingénieur de l'Ecole Polytechnique d'Alger, il a obtenu son diplôme de doctorat à l'Université Pierre et Marie Curie Jussieu (PARIS VI) en janvier 1994. Ses travaux de recherche concernent principalement la conception et la modélisation des dispositifs électromagnétiques non conventionnels.

Bernard MULTON, Professeur des universités à l'antenne de Bretagne de l'Ecole Normale Supérieure de Cachan, Directeur du département de mécatronique, est co-responsable de l'équipe "Conception d'actionneurs » du LESiR (UPRESA CNRS). Il est agrégé de Génie Electrique (1982), titulaire d'un doctorat (Paris VI 1985) et d'une habilitation à diriger des recherches (1994). Ses domaines de recherche concernent la conception de machines électriques spéciales (réluctance variable et aimants) à alimentation électronique ainsi que la production décentralisée d'électricité d'origine renouvelable. 
24 Revue Internationale de Génie Electrique.

Nicolas BERNARD est agrégé de Génie Electrique (1997), ancien élève de l'Ecole Normale Supérieure de Cachan. Il prépare, depuis 1998 à l'antenne de Bretagne du LESiR, une thèse de doctorat de l'ENS de Cachan sur le thème "Etude et alimentation d'un moteurgénérateur pour le stockage électromécanique de l'énergie ». 